\title{
Platelet-in-Box Colloidal Quantum Wells: CdSe/CdS@CdS Core/Crown@Shell Heteronanoplatelets
}

\author{
Yusuf Kelestemur, Burak Guzelturk, Onur Erdem, Murat Olutas, Kivanc Gungor, \\ and Hilmi Volkan Demir*
}

Here, the CdSe/CdS@CdS core/crown@shell heterostructured nanoplatelets (NPLs) resembling a platelet-in-box structure are developed and successfully synthesized. It is found that the core/crown@shell NPLs exhibit consistently substantially improved photoluminescence quantum yield compared to the core@shell NPLs regardless of their CdSe-core size, CdS-crown size, and CdS-shell thickness. This enhancement in quantum yield is attributed to the passivation of trap sites resulting from the critical peripheral growth with laterally extending CdS-crown layer before the vertical shell growth. This is also verified with the disappearance of the fast nonradiative decay component in the core/crown NPLs from the time-resolved fluorescence spectroscopy. When compared to the core@shell NPLs, the core/crown@shell NPLs exhibit relatively symmetric emission behavior, accompanied with suppressed lifetime broadening at cryogenic temperatures, further suggesting the suppression of trap sites. Moreover, constructing both the CdS-crown and CdS-shell regions, significantly enhanced absorption cross-section is achieved. This, together with the suppressed Auger recombination, enables the achievement of the lowest threshold amplified spontaneous emission $\left(\approx 20 \mu \mathrm{cm}^{-2}\right)$ from the core/crown@shell NPLs among all different architectures of NPLs. These findings indicate that carefully heterostructured NPLs will play a critical role in building high-performance colloidal optoelectronic devices, which may even possibly challenge their traditional epitaxially grown thin-film based counterparts.
Y. Kelestemur, B. Guzelturk, O. Erdem, Dr. M. Olutas,

K. Gungor, Prof. H. V. Demir

Department of Electrical and Electronics Engineering Department of Physics

UNAM - Institute of Materials Science

and Nanotechnology

Bilkent University

Ankara 06800, Turkey

E-mail:volkan@bilkent.edu.tr, hvdemir@ntu.edu.sg

Dr. M. Olutas

Department of Physics

Abant Izzet Baysal University

Bolu 14280, Turkey

Prof. H. V. Demir

Luminous! Center of Excellence for Semiconductor

Lighting and Displays

School of Electrical and Electronic Engineering

School of Physical and Materials Sciences

School of Materials Science and Nanotechnology

Nanyang Technological University

Singapore 639798, Singapore

DOI: $10.1002 / \mathrm{adfm} .201600588$

\section{Introduction}

Thanks to recent advancements in the science of colloidal synthesis within the past 20 years, different types of semiconductor nanocrystals have been synthesized with great control over their size, shape, and composition. This started with the synthesis of highly monodisperse spherical nanocrystals ${ }^{[1]}$ and continued with more complex and anisotropic structures including rods ${ }^{[2]}$ and tetrapods. ${ }^{[3]}$ Recently, it has been shown that free-standing colloidal nanoplatelets (NPLs) having lateral dimensions on the order of tens of nanometers and magic-sized vertical thicknesses can be also synthesized with colloidal routes. ${ }^{[4]}$ Owing to pure tight confinement along their vertical thickness, the NPLs exhibit strongly thicknessdependent electronic structure and optical properties. ${ }^{[5,6]}$ With the synthesis of NPLs having atomically-flat surfaces, inhomogeneous broadening is almost suppressed and extremely narrow emission bandwidths $(\Delta \mathrm{E} \sim 35 \mathrm{meV}$ at room temperature) can be achieved. In addition, when compared to semiconductor

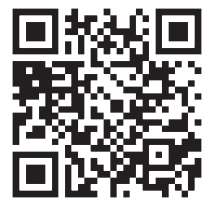
nanocrystals having different geometry and shape, the NPLs feature ultrafast fluorescence lifetime due to their giant oscillator strength ${ }^{[5]}$ and large linear and nonlinear absorption cross-sections, ${ }^{[7,8]}$ which are highly crucial for achieving low-threshold lasing.

With all of these appealing properties, the NPLs have become highly promising for next-generation solution-processed optoelectronic device applications including lightemitting-diodes (LEDs) and lasers. However, similar to other classes of colloidal semiconductor nanocrystals, ${ }^{[9,10]}$ core-only NPLs have suffered from the poor stability owing to their large surface area that result in lower photoluminescence quantum yield (PL-QY). ${ }^{[11]}$ The stability issues and low PL-QYs become more dramatic especially for the solid films of these NPLs since they have tendency to form needle-like stacks ${ }^{[12]}$ due to strong van der Walls forces. For these stacked NPLs, strongly quenched photoluminescence has been reported arising from ultraefficient exciton transport assisted hole trapping. ${ }^{[13]}$ 
Up to now, to enhance stability and PL-QY of NPLs, core/ crown (laterally grown shell) ${ }^{[14-18]}$ and core@shell (vertically grown shell ${ }^{[19,20]}$ architectures have been synthesized and studied intensively. Among different varieties of core/crown (C/C) NPLs, CdSe/CdS C/C NPLs have been one of the most widely studied material systems. Since CdS-crown layer is grown only in the lateral direction, the confinement in the vertical thickness is not changed and thus there is no noticeable change observed in the emission spectrum of CdSe/CdS C/C NPLs as compared to the CdSe core-only NPLs. However, with the passivation of trap sites originating from the sidewall of core-only NPLs, PL-QY can be greatly increased in the C/C NPLs. Also, with the formation of CdS-crown layer, absorption cross-section in the near-UV and blue spectral ranges is substantially enhanced due to excitonic absorption in the larger band gap CdS. Furthermore, the CdS-crown layer acts as a light-harvesting antenna, where the excitons photogenerated in the CdS-crown layer are rapidly transferred to the CdSe-core region, and makes contribution to the core emission. Therefore, with their enhanced optical properties, CdSe/CdS C/C NPLs have been shown to boost the optical gain performance in the NPLs. ${ }^{[21]}$ However, due to lack of proper passivation on their larger lateral surfaces, they still suffer from serious stability issues.

Also, recently with the synthesis of core@shell (C@S) architectures, the optical properties of NPLs have been shown to be enhanced with respect to the core-only NPLs since exciton is further confined within the core of the heterostructure and away from the lateral surface traps and possible dangling bonds. ${ }^{[19]}$ Generally, shell growth in NPLs has been carried out using two different approaches: one-pot synthesis ${ }^{[19]}$ and colloidal atomic-layer-deposition (c-ALD) technique. ${ }^{[20]}$ In both cases, with the formation of shell layers, a significant amount of spectral redshift is observed in the absorption and emission spectra depending on the thickness and composition of the shell layers. This arises due to the relaxing vertical confinement of the electron and holes (thus, excitons) and changing the dielectric medium in the C@S NPLs. Although a significant amount of broadening of the emission is also observed in the C@S NPLs with respect to the core-only NPLs, the C@S architecture still features narrower emission bandwidths when compared to other class of colloidal semiconductor nanocrystals including quantum dots and nanorods. ${ }^{[22]}$ Also, a higher level of photoluminescence quantum yield (up to $80 \%$ ) is achieved for CdSe@CdS C@S NPLs synthesized with one-pot synthesis. ${ }^{[22]}$ In addition,CdSe@CdS C@S NPLs, due to the separation of electron and hole wave functions, exhibit suppressed Auger recombination $(\mathrm{AR})^{[23]}$ with reduced blinking behavior ${ }^{[22]}$. With their promising properties, light-emitting diodes having narrower emission bandwidths ${ }^{[24]}$ and optically pumped lasers with very low threshold ${ }^{[25]}$ have been demonstrated by using NPLs in the C@S architecture. However, due to the formation of trap sites during the shell growth, the performance of C@S NPLs has been shown to be limited. ${ }^{[22]}$ Therefore, to achieve further enhancement in the excitonic properties of NPLs, newly designed architectures are highly welcomed.

Here, we have developed and demonstrated the synthesis of CdSe/CdS@CdS core/crown@shell (C/C@S) NPLs resembling a platelet-in-box structure and report their systematic characterization. Starting with the CdSe-core NPLs, a crown layer of CdS is grown only in the lateral directions. In these CdSe/CdS C/C NPLs, with the passivation of periphery of the starting CdSe-core NPLs, a faster nonradiative decay component ( $\approx 0.15 \mathrm{~ns}$ ) originating from the fast hole trapping is eliminated, which results in enhanced PL-QY (up to $90 \%$ ). Then, by using colloidal atomic-layer-deposition technique, smooth CdS shell layers are deposited on both the core-only and C/C NPLs with atomically precise shell thickness control. With the formation of CdS shells, in both cases, a large redshift is observed in the absorption and emission spectra along with the spectral broadening of the excitonic features. When compared to C@S NPLs, C/C@S NPLs exhibit significantly enhanced absorption cross-section in the blue spectral region owing to CdS-crown layer and also improved PL-QY due to better passivation of the sidewalls of the inner CdSe-core. In addition, amplitude-averaged fluorescence lifetimes of the C/C@S NPLs are found to be increased as a function of the increasing CdS shell thickness, which can be well explained by the delocalization of electron wavefunctions into the CdS shell layer leading to a quasi TypeII electronic structure. On the other hand, in the C@S NPLs, amplitude-averaged lifetimes are observed to remain almost constant as a function of the CdS shell thickness, which is attributed to the competition between the elongated radiative decay due to quasi Type-II character and increased nonradiative decay due to the created traps during shell growth. Suppression of trap sites in the C/C@S NPLs has been further evidenced by temperature-dependent photoluminescence study and timeresolved fluorescence spectroscopy (TRF). Lifetime broadening effect (observed for different lifetimes in the low energy side and also in the high energy side of the emission peak) is considerably suppressed in the C/C@S NPLs as compared to that in the C@S NPLs. Finally, with their enhanced optical properties, the C/C@S NPLs achieve higher optical gain performance as compared to the C@S and C/C NPLs. Optical gain threshold as low as $\approx 20 \mu \mathrm{J} \mathrm{cm}^{-2}$ is shown thanks to the increased PL-QY, enhanced absorption cross-section and eliminated trap sites in the C/C@S NPLs.

\section{Results and Discussion}

In this study, CdSe/CdS@CdS core/crown@shell (C/C@S) NPLs having a novel 3D architecture have been synthesized in three separate steps. First, we started with the synthesis of CdSe core NPLs having four complete monolayers (4 ML) of CdSe with an additional layer of $\mathrm{Cd}$ atoms by using a slightly modified recipe from the literature (see the Experimental Section for details). ${ }^{[14]}$ When compared to CdSe NPLs having different vertical thicknesses, these CdSe core NPLs have been preferred in this study because of their optimized synthesis condition and higher stability. Absorption and PL emission spectra of these CdSe-core NPLs are given in Figure 1a. With their quantum well-like electronic structure, the sharp excitonic features around 480 and $511 \mathrm{~nm}$ corresponding to electron/light-hole and electron/heavy-hole transitions, respectively, are clearly observed from the absorption spectrum. Also, they feature narrower emission linewidth $(\approx 8 \mathrm{~nm})$ with a PL-QY of $\approx 30 \%$. From TEM images (see the 

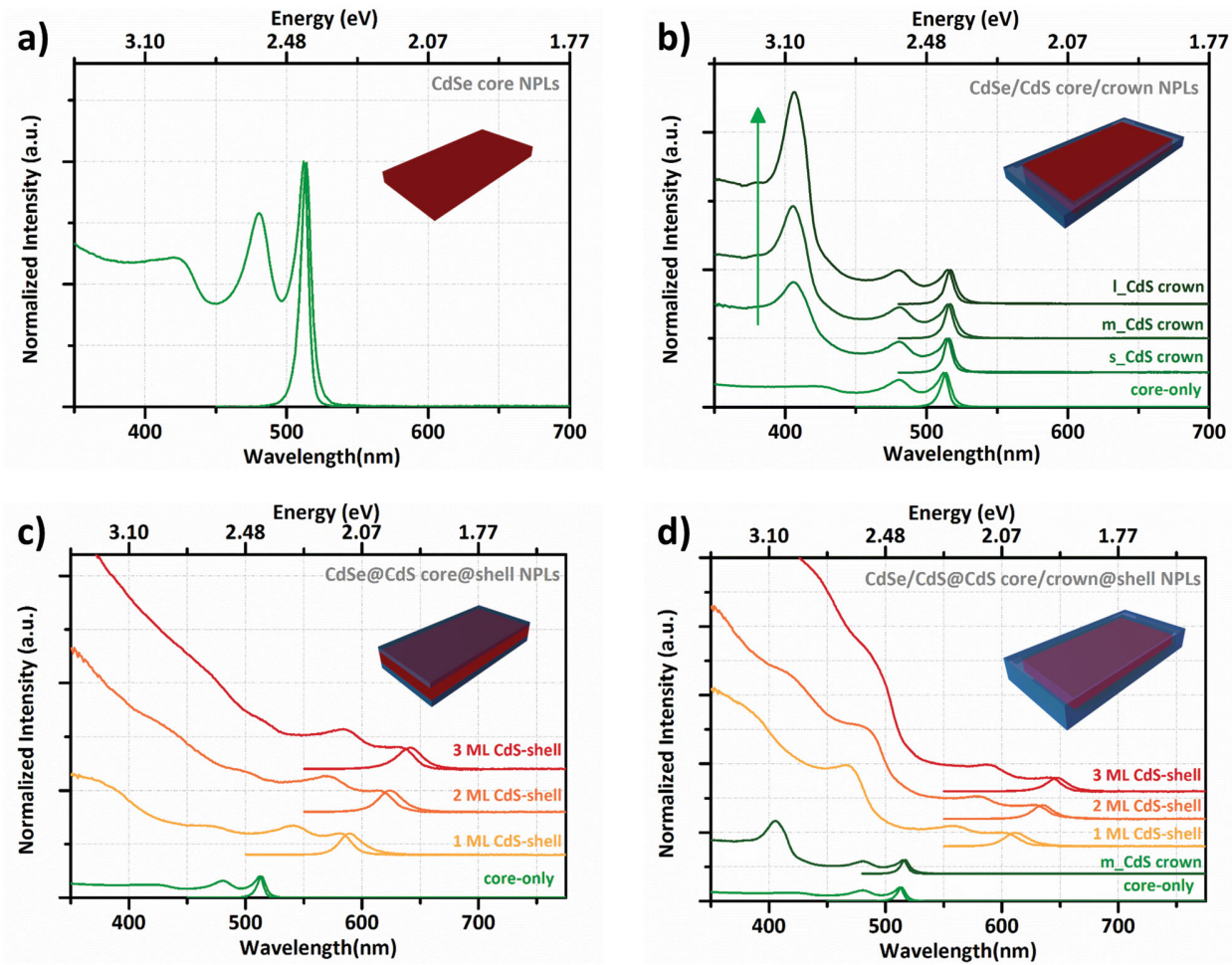

Figure 1. Absorption and photoluminescence spectra of a) CdSe core-only NPLs, b) CdSe/CdS core/crown NPLs having different crown sizes, c) CdSe@CdS core@shell, and d) CdSe/CdS@CdS core/crown@shell NPLs with varying CdS shell thicknesses. Here, the CdSe/CdS core/crown NPLs samples having small, medium, and large CdS crown sizes are labeled as s_CdS crown, m_CdS crown, and I_CdS crown, respectively.

Supporting Information), formation of almost square-shaped CdSe core-only NPLs (with lateral dimensions of $11.1 \pm 1.4$ $\mathrm{nm}$ and $14.4 \pm 1.7 \mathrm{~nm}$ ) can be clearly seen. These core NPLs have been used as a seed for the subsequent synthesis of core/crown (C/C), core@shell (C@S) and core/crown@shell (C/C@S) architectures.

In the second step, CdSe/CdS C/C NPLs have been synthesized using core-seeded approach following a previously published procedure with slight modifications (see the Experimental Section). ${ }^{[14]}$ By using cadmium acetate as a cadmium precursor, anisotropic growth of CdS layer is achieved and CdS-crown layer is grown only in the lateral direction. Also, varying the amount of injected solution, CdSe/CdS C/C NPLs with different crown sizes have been prepared. As it can be seen from Figure $1 \mathrm{~b}$, with the formation of small CdS-crown layer, a new absorption peak around $\approx 406 \mathrm{~nm}$ becomes apparent corresponding to the band gap of $\mathrm{CdS}$ NPLs having the same thickness with CdSe core NPLs. With the increased size of the CdS-crown layer, this absorption peak becomes more pronounced and significantly enhances the absorption cross-section of the resulting NPLs in the blue spectral region. Also, by using photoluminescence excitation spectroscopy (PLE) (see the Supporting Information), it is shown that this CdS-crown layer acts as an antenna, where excitons formed in the CdS-crown region are transferred to the CdSe-core region, and makes a major contribution to the emission. On the other hand, owing to the only lateral growth of CdS-crown layer, no significant change is observed in the excitonic features of the CdSe core NPLs. The slight redshift $(\approx 3-4 \mathrm{~nm})$ in the absorption and emission peaks is attributed to the change of dielectric constants due to the formation of the CdS-crown layer. In addition, with the passivation of the periphery of the CdSe core NPLs, a substantially higher PL-QY (up to $90 \%$ ) is obtained depending on the size of the CdS-crown layer. While the CdSe/CdS C/C NPLs having a narrower CdS-crown width suffer from the incomplete and/ or uneven growth of the CdS-crown layer, the CdSe/CdS C/C NPLs having a wide enough layer suffer from the formation of cracks, which may act as a trap site. With the addition of a small amount of oleic acid, the stability and quality of the CdS-crown layer can be greatly improved when compared to our previous synthesis. Nevertheless, it is not easy to achieve sharp boundaries in CdSe/CdS C/C NPLs similar to CdSe/ CdTe C/C NPLs. ${ }^{[17,18]}$ Therefore, optimized CdS-crown layer coating is essential to achieve high optical performance from CdSe/CdS C/C NPLs.

In the last step, CdS-shell coating has been performed for both the CdSe-core only and the CdSe/CdS C/C NPLs having different crown sizes by using c-ALD technique. ${ }^{[20]}$ As compared to the CdS-shell growth with one-pot synthesis, c-ALD offers great advantages including the formation of smooth CdSshell layer with atomically precise shell thickness control. As it can be seen from Figure 1c,d, with the formation of CdS-shell, a significant amount of redshifting in the absorption and emission spectra has been observed for both the core@shell (C@S) and the core/crown@shell (C/C@S) NPLs. This strongly redshifting behavior can be explained with the growth of CdS-shell in the vertical direction, making the initially very tight quantum 
confinement relatively relaxed. Owing to quasi-Type-II like band alignment between CdSe and CdS material systems, electrons can spread across the whole structure (throughout both the $\mathrm{CdSe}$-core and CdS-shell regions), while holes are mostly confined to the CdSe-core region. Therefore, with the separation of electron and hole wavefunctions, strongly redshifted emission can be obtained. Also, when compared to the C@S NPLs having the same CdS-shell thickness, the C/C@S NPLs exhibit an additional redshifting in their absorption and emission spectra. For example, while the C@S NPLs with one-monolayer (1 ML) CdS-shell thickness dissolved in hexane have an emission peak around $\approx 589 \mathrm{~nm}$, the C/C@S NPLs with the same CdS-shell thickness possess its emission peak around $\approx 610 \mathrm{~nm}$.

This additional redshifting in the C/C@S NPLs could possibly be originated from two-potential sources: a nonuniform CdS-shell coating and/or change in dielectric constant, which is known to be effective. Therefore, to check out the first hypothesis, transmission electron microscopy (TEM) and photoluminescence excitation spectroscopy (PLE) have been performed. From the HAADF-TEM images (Figure S7, Supporting Information), the thickness of the CdSe/CdS@CdS core/crown@shell NPLs having $2 \mathrm{ML} \mathrm{CdS}$ shell is determined as $\approx 2.7 \mathrm{~nm}$, which is matched with the estimated thickness of $\approx 2.5 \mathrm{~nm}$ (see the Supporting Information). Also, the PLE spectra taken at the lower energy side, the higher energy side and the peak of the emission exhibit the same excitonic transitions, which suggest that there is no considerable inhomogeneous broadening. This rules out the possibility of CdS-shell thickness variations and thus the nonuniform CdS-shell coating of the C/C@S NPLs is not possible because variation in shell-thickness would cause NPL sub-populations with varying excitonic transitions, which would result in an inhomogeneous broadening in the emission and absorbance spectra (see the Supporting Information). Therefore, this additional redshifting in the C/C@S NPLs is explained with the difference in the effective dielectric constant. With the encapsulation of the CdSe-core region by the CdS-crown and CdS-shell regions leading to a platelet-in-box structure, the dielectric constant of the C/C@S NPLs has indeed been significantly increased when compared to the C@S NPLs, resulting in additional redshifting in absorption and emission spectra. Also, similar trends observed between the CdSe-core only NPLs and the CdSe/CdS core/crown NPLs further suggest that the additional shifting from C@S to the C/C@S NPLs originate from the difference in the dielectric constant (see Figure 1b).

In addition to strongly redshifted light-hole and heavy-hole transitions with the emission peak, broadening of the emission bandwidths in the C@S and C/C@S NPLs has been observed when compared to the CdSe-core only NPLs. While the CdSe-core only NPLs exhibit full-width-at-half-maximum (FWHM) of $\approx 35-40 \mathrm{meV}$, the C@S and C/C@S NPLs have FWHM of $\approx 62-90 \mathrm{meV}$. This broadening was previously reported in the C@S NPLs regardless of the shell deposition method and attributed to the enhanced exciton-phonon coupling upon the deposition of CdS-shell. ${ }^{[22]}$ Also, we have observed that structural quality of the seed NPLs (either the CdSe-core or the $\mathrm{CdSe} / \mathrm{CdS} \mathrm{C} / \mathrm{C} \mathrm{NPLs}$ ) is responsible for the broadening of emission bandwidth. Depending on the quality of seed NPLs, the FWHM of the C@S, and C/C@S NPLs ranges from $\approx 62$ to $90 \mathrm{meV}$. For example, while the
CdSe/CdS@CdS C/C@S NPLs having medium CdS crown size exhibit FWHM of $\approx 62 \mathrm{meV}$, the CdSe@CdS C@S NPLs exhibit FWHM of $\approx 76 \mathrm{meV}$. The decreased broadening in the PL emission of the C/C@S NPLs having medium crown size can be explained with the passivation of trap sites in the CdSecore only NPLs. On the other hand, the C/C@S NPLs having smaller and larger crown sizes exhibit broader PL emission bandwidths ( $\approx 90 \mathrm{meV}$ ) when compared to the C@S NPLs due to incomplete and/or uneven growth of CdS-crown layer (for the smaller crown) and formation of cracks in the CdS-crown layer (for the larger crown).

We have also measured the PL-QY of both the C@S and C/C@S NPLs by using relative PL-OY measurement technique with Rhodamine 6G. Up to now, we have synthesized different C@S and C/C@S NPLs by using various CdSe-core and CdScrown sizes. Regardless of the CdSe-core size, CdS-crown size and CdS-shell thickness, the C/C@S NPLs are found to be consistently exhibiting higher PL-QY (up to $\approx 40 \%$ ) than that of the C@S NPLs. Also, with increasing the CdS-shell thickness, decreasing of the PL-QY is observed for both the C@S and the C/C@S NPLs. This decreasing trend of PL-QY with increasing the shell thickness can possibly be attributed to our shell deposition method, which was performed at room temperature under ambient atmosphere, resulting in the formation of defect sites. For example, the PL-QY of CdSe/CdS@CdS C/C@S NPLs (with medium crown size) having 1 and 3 ML CdS-shell thickness are $\sim 36 \%$ and $\approx 18 \%$, respectively. Similarly, for the CdSe@CdS C@S NPLs having 1 and 3 ML CdSshell thickness are $\approx 20 \%$ and $\approx 8 \%$, respectively. The increased PL-QY of the C/C@S NPLs with respect to the C@S NPLs has shown the importance of sidewall passivation of the CdSe-core NPLs.

To develop a better understanding of the excitonic properties of the CdSe-core based hetero-NPLs in different architectures including CdSe/CdS C/C, CdSe@CdS C@S, and CdSe/CdS@CdS C/C@S NPLs, we performed time-resolved fluorescence (TRF) spectroscopy. In-solution samples of NPLs (dissolved in hexane), which were gently cleaned, are used for TRF measurements to avoid stacking issue. Previously, it has been shown that NPLs have tendency to form columnlike stacks depending on concentration of NPL solution, ligand concentration, and cleaning procedures. ${ }^{[11,12,21]}$ In the stacked NPL assemblies, ultrafast energy transfer among the NPLs ${ }^{[26]}$ assists hole-trapping, which alters excitonic properties including significantly accelerated overall fluorescence decay rates with almost completely quenched photoluminescence. ${ }^{21]}$ Therefore, sample quality is highly crucial to correctly analyze resulting excitonic properties of different architectures of hetero-NPLs.

Fluorescence decay curves of our CdSe-core only NPLs, CdSe/CdS C/C NPLs having different crown sizes, CdSe@ CdS C@S and CdSe/CdS@CdS C/C@S NPLs with different shell thicknesses are presented in Figure 2. These decay curves are analyzed by decay functions, which are sum of exponential decays, resulting in reduced $\chi^{2}$ about 1 with uniform residuals. For the CdSe-core only NPLs, a multiexponential decay is observed with an amplitude-averaged fluorescence lifetime $\left(\tau_{\text {av }}\right)$ of $\approx 1.86 \mathrm{~ns}$. This multiexponential decay kinetics of the CdSe-core only NPLs has been previously 
a)

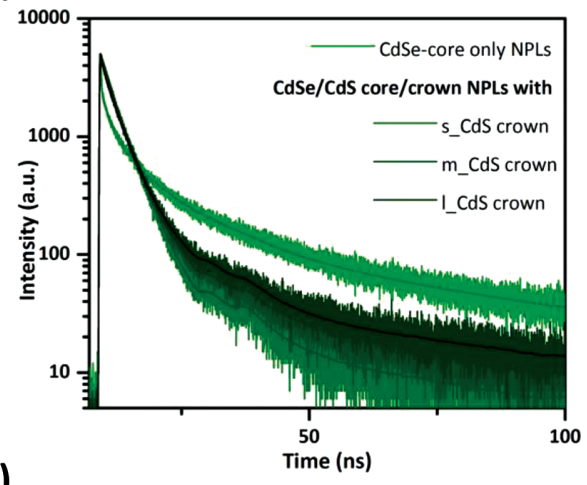

c)

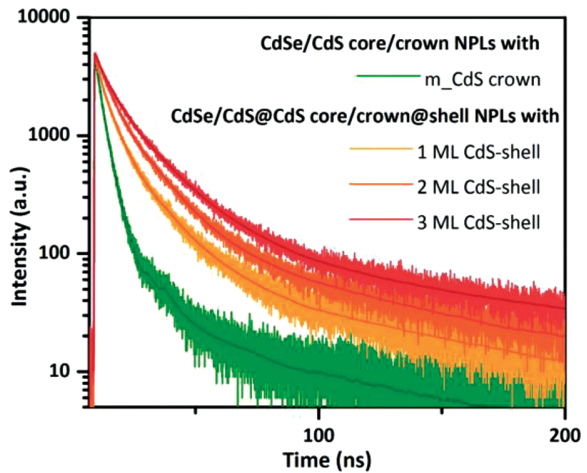

b)

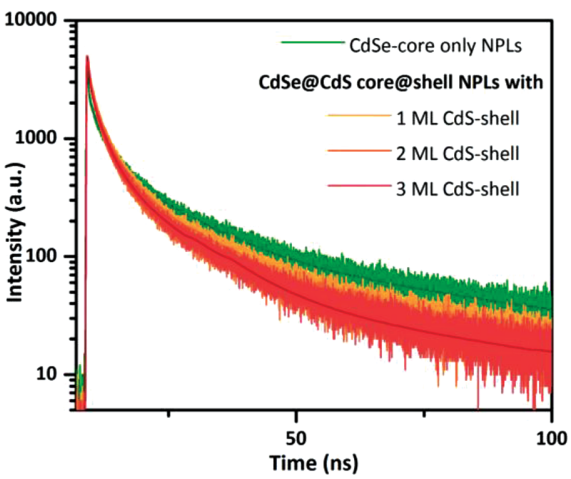

d)

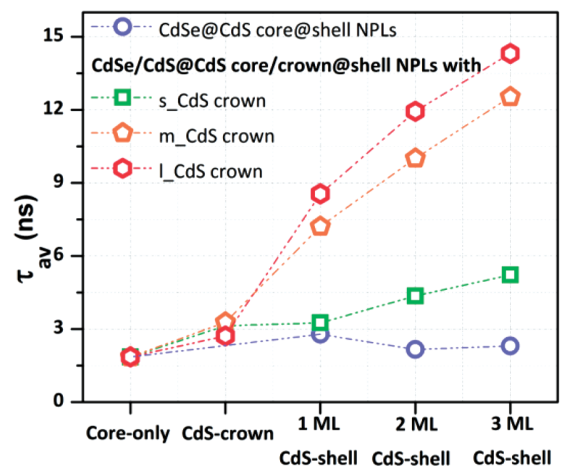

Figure 2. Time-resolved fluorescence (TRF) spectroscopy measurements of a) CdSe-core only NPLs and CdSe/CdS core/crown NPLs with varying CdS-crown size, b) CdSe@CdS core@shell NPLs, and c) CdSe/CdS@CdS core/crown@shell NPLs with different CdS-shell thicknesses.d) Amplitudeaveraged fluorescence lifetime of the core, core/crown, core@shell, and core/crown@shell NPLs having different CdS-crown sizes as a function of the shell thickness.

observed from both single and ensemble measurements and attributed to the presence of several radiative decay channels in NPLs. ${ }^{[7,27]}$ Although it is hard to interpret these distinct lifetime components one by one, the fastest lifetime component $(\approx 0.15 \mathrm{~ns})$ is attributed to the nonradiative decay pathway originating from the fast hole-trapping due to incomplete passivation of $\mathrm{Cd}$ atoms on the surface. ${ }^{[28,29]}$ Also, with increasing the lateral size of CdSe core-only NPLs, the contribution of the fastest lifetime component has been shown to become more pronounced accompanied with decreased PL-QY. ${ }^{[7]}$ This finding strongly supports the discussions above and shows the importance of peripheral passivation of sidewalls of the core-only NPLS.

With the formation of the CdS-crown layer (only in the lateral direction), amplitude-averaged fluorescence lifetimes are slightly increased to 3.13 and $3.28 \mathrm{~ns}$ for having smaller and medium crown sizes, respectively. As it can be seen from Figure $2 \mathrm{a}$, with the sidewall passivation of CdSe core-only NPLs, the fastest decay component completely disappears. This also explains the much higher PL-QY (up to 90\%) of the CdSe/ CdS C/C NPLs with respect to the CdSe core-only NPLs. On the other hand, with the formation of larger CdS-crown size, we start to observe the fastest lifetime component $(\approx 0.36 \mathrm{~ns})$ again due to the formation of cracks with continuing crown growth. Therefore, there is an optimum crown size in the NPLs, as discussed previously. ${ }^{[21]}$ However, the CdSe/CdS C/C NPLs having larger crown size still exhibit longer $\tau_{\text {av }}$ of $\approx 2.72 \mathrm{~ns}$ with respect to the CdSe core-only NPLs ( $\approx 1.86 \mathrm{~ns})$. This slightly elongation in $\tau_{\mathrm{av}}$ of the CdSe/CdS C/C NPLs can again be attributed to the change in the effective dielectric constant with the formation of CdS-crown layer. Since the CdS-crown layer is grown only in the lateral direction, this does not cause any change in the tight vertical confinement.

With the growth of CdS-shell (in the vertical direction), increased $\tau_{\mathrm{av}}$ 's are observed for the CdSe/CdS@CdS C/C@S NPLs. This increasing trend of $\tau_{\mathrm{av}}$ is explained with the delocalization of electrons into the CdS-shell region owing to the lower energy barrier for electrons between CdSe and $\mathrm{CdS}$ material systems. Also, with increasing thickness of the CdS-shell layer, the separation of electron and hole wavefunctions is further increased, resulting in an elongated amplitude-averaged fluorescence lifetime. In addition, the increased CdS-crown size in the CdSe/CdS@CdS C/C@S NPLs results in further elongated $\tau_{\mathrm{av}}$. For example, while $\tau_{\mathrm{av}}$ of the C/C@S NPLs with small lateral size increases from 3.13 (for the CdSe/CdS C/C NPLs) to $5.23 \mathrm{~ns}$ (for the $3 \mathrm{ML}$ CdSe/CdS@CdS C/C@S NPLs), those of the C/C@S NPLs with medium crown size increase from 3.28 (for the CdSe/CdS C/C NPLs) to $12.54 \mathrm{~ns}$ (for the CdSe/ CdS@CdS C/C@S NPLs with 3ML CdS shell) owing to the uniform coating of CdS-crown layer and complete passivation of CdSe core sidewalls. On the other hand, $\tau_{\mathrm{av}}$ of the CdSe@ CdS C@S NPLs remains almost constant upon the growth of CdS-shell. This can possibly be explained with the competition between the elongated radiative decay components owing 
a)

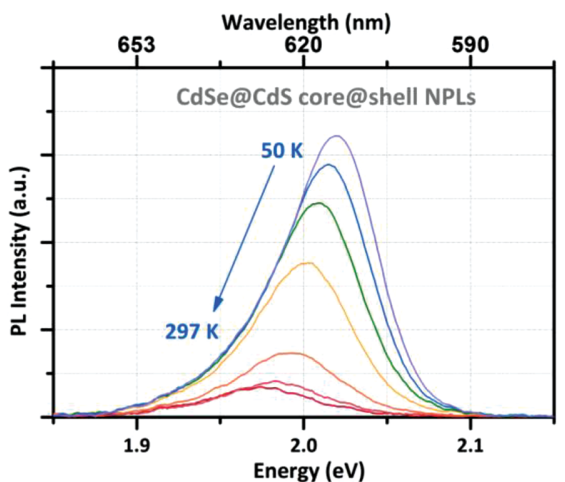

c)

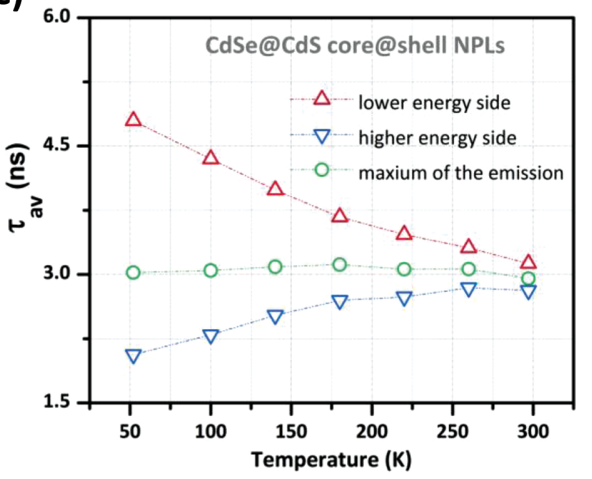

b)

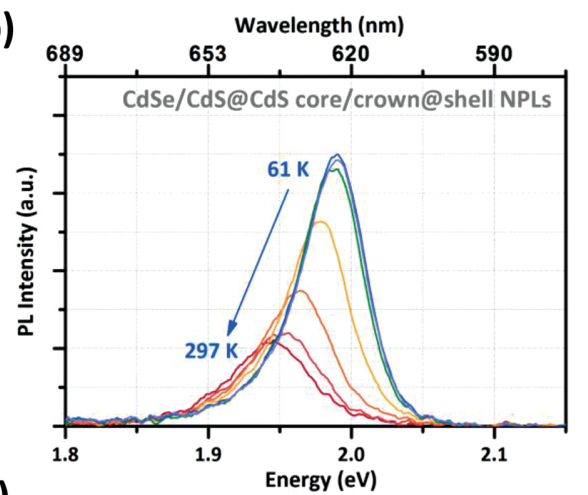

c)

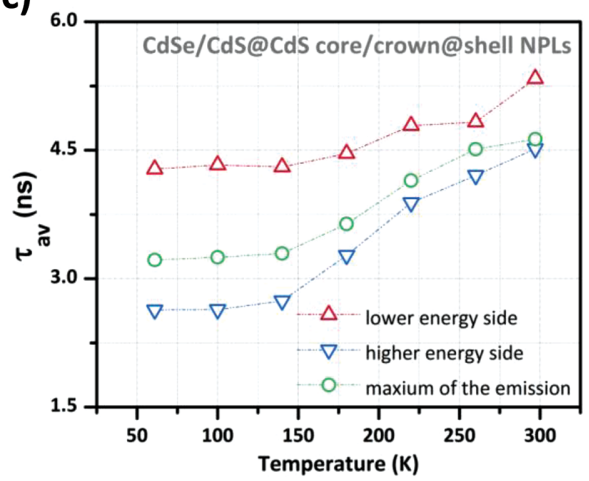

Figure 3. Temperature-dependent PL spectra of a) CdSe@CdS core@shell NPLs and b) CdSe/CdS@CdS core/crown@shell NPLs having2 ML CdSshell thicknesses. Amplitude-averaged fluorescence lifetimes ( $\left.\tau_{\mathrm{av}}\right)$ of these c) core@shell and d) core/crown@shell NPLs at the lower energy side, at the higher energy side and at the maximum of emission.

to the formation of CdS-shell and the fast nonradiative decay components due to the trap formation as a result of the shell growth. The decreasing PL-QY also strongly supports the increased nonradiative decay in the CdSe@CdS C@S NPLs with increasing CdS-shell thickness.

To provide further insight into the emission kinetics of the CdSe@CdSC@S and the CdSe/CdS@CdS C/C@S NPLs, we performed temperature-dependent PL spectroscopy. Temperature-dependent PL spectra of these CdSe@CdS C@S and CdSe/CdS@CdS C/C@S NPLs (having 2 ML CdS-shell thicknesses) are given in Figure 3a,b, respectively. For both of the samples, we observe decreased intensity of the photoluminescence with increasing temperature. Such quenching in the emission has also previously been observed in different classes of semiconductor nanocrystals and attributed to the surface/interface defects, which act as carrier-trapping centers at higher temperatures, resulting in nonradiative recombination and decreasing the photoluminescence. ${ }^{[30]}$ Therefore, strongly quenched emission with increasing temperature can be explained with the existence of a large number of trap sites. In our study, we observed that, at297 K the CdSe@CdS C@S NPLs maintain $\approx 11.5 \%$ of their initial photoluminescence intensity measured at $50 \mathrm{~K}$, while the CdSe/CdS@CdS C/C@S NPLs sustain $\approx 32.2 \%$ for the same temperature range. This indicates that in the core/crown@shell NPLs, the PL-QY is better preserved at high temperatures as compared to the core@ shell NPLs, as another advantages of the C/C@S heterostructure. Thus, the better preservation of photoluminescence with increasing temperature in the CdSe/CdS@CdS C/C@S NPLs may suggest reduced surface/interface defects with respect to those of the CdSe@CdS C@S NPLs owing to the additional passivation of CdSe core periphery with CdS crown layer.

Also, another significant point observed in the temperaturedependent steady-state PL spectra of the CdSe@CdS C@S and the CdSe/CdS@CdS C/C@S NPLs is their asymmetric emission profile, which can be observed even at room temperature. With decreasing temperature, their asymmetric behavior becomes more pronounced with a long tail on the lower energy side of the emission (see Figure 3a). To unravel this asymmetric emission behavior, we performed temperature-dependent TRF spectroscopy at the lower energy side, at the higher energy side and at the emission peak. $\tau_{\mathrm{av}}{ }^{\prime}$ s of the C@S and C/C@S NPLs at different temperatures are shown in Figure $3 \mathrm{c}, \mathrm{d}$, respectively. Unlike the CdSe-core only NPLs exhibiting accelerated decay rate with decreasing temperature, $\tau_{\mathrm{av}}$ of the CdSe@CdS C@S NPLs remains almost the same with decreasing temperature. On the other hand, when we analyze $\tau_{\text {av }}$ in the lower and higher energy sides of the emission peak, different trends are observed with decreasing temperature. While $\tau_{\mathrm{av}}$ is continuously increasing from 3.13 to $4.80 \mathrm{~ns}$ at the lower energy side as a function of the decreasing temperature, the opposite behavior is obtained for the lifetime measured for the higher energy side ( $\tau_{\text {av }}$ decreasing from 2.81 to $2.06 \mathrm{~ns}$ ).

This kind of asymmetric emission along with significant lifetime broadening has also been observed in epitaxially grown thin-film quantum wells and explained with the charge carrier localization in the active traps sites, which can be steam from to the surface/interface defects and strain formation. ${ }^{[31-33]}$ Owing 
to charge carrier localization in the trap sites, emission from these trap sites originate at a lower energy than the bandgap energy accompanied with longer radiative lifetimes. Recently, similar behavior has been also observed by Dubertret group in the CdSe@CdS C@S NPLs and attributed to the formation of defects upon the shell deposition and surface defects. ${ }^{[22]}$ Here, we demonstrate that in contrast to the CdSe@CdS C@S NPLs, the CdSe/CdS@CdS C/C@S NPLs exhibit less asymmetric emission with suppressed lifetime broadening, which we attribute to the enhanced passivation of the CdSe core-only NPLs and the reduced number of traps sites with the formation of CdS crown layer. As it can be seen from Figure 3d, by decreasing the temperature, accelerated decay rates are achieved from both the lower and higher energy sides of the emission. With all of these important findings, it can be concluded that, with the formation of the CdS-crown layer, the interface/surface defects are suppressed and favorable optical properties can be achieved by the proposed core/crown@shell architecture.

Recently, colloidal NPLs have been shown to be highly promising for low threshold lasing owing to their suppressed Auger recombination (AR), giant oscillator strength and increased absorption cross-section, enabling high performance all-colloidal nanocrystal lasers. ${ }^{[7,21,25,34-36]}$ Also, when compared to other classes of colloidal semiconductor nanocrystals, their higher gain coefficients, broader gain bandwidth, and longer gain lifetime make them highly favorable for practical lasing applications. ${ }^{[34]}$ It has been demonstrated that even simple CdSe core-only NPLs having different thicknesses surpass the optical gain performance levels of custom designed colloidal nanocrystals emitting in the same spectral range. For example, 3 ML CdSe core-only NPLs having emission in the blue spectral region with a lower PL-QY $(\approx 10 \%)^{[34]}$ exhibit desirably lower gain thresholds than carefully engineered $\mathrm{Cd}_{\mathrm{x}} \mathrm{Zn}_{1-\mathrm{x}} \mathrm{S} / \mathrm{ZnS}$ core/ shell quantum dots having smooth interface with almost nearunity PL-QY. ${ }^{[37]}$ This is mainly facilitated by the large absorption cross-section of the NPLs. Furthermore, the absence of inhomogeneous broadening also allows for enhanced optical gain performance with reduced losses due to the intrinsic absorption over a wider spectral range. In addition, it has been demonstrated that by using the CdSe/CdS core/crown ${ }^{[21]}$ and CdSe@CdS core@shell[25] architectures, further better optical gain performance can be achieved thanks to their increased absorption cross-section and suppressed Auger recombination (AR) with respect to the CdSe core-only NPLs. In this study, to achieve even further enhanced properties, we studied systematically optical gain performances of CdSe/CdS@CdS core/ crown@shell NPLs along with the CdSe core-only, CdSe/CdS core/crown and CdSe@CdS core@shell NPLs.

For the optical gain measurements, highly close-packed and uniform films were prepared on bare quartz substrates with spin coating of the concentrated solutions of NPLs. The samples were excited with a femtosecond laser beam having a wavelength of $400 \mathrm{~nm}$ (providing $120 \mathrm{fs}$ laser pulses at a $1 \mathrm{kHz}$ repetition rate) by using stripe-excitation geometry. The laser beam was focused (over an excitation area of $\approx 200 \mu \mathrm{m}$ by $5 \mathrm{~mm}$ as measured by an optical profilometer, Newport) on the samples by using a cylindrical lens. The collected PL spectra for the samples are presented in Figure 4. For the
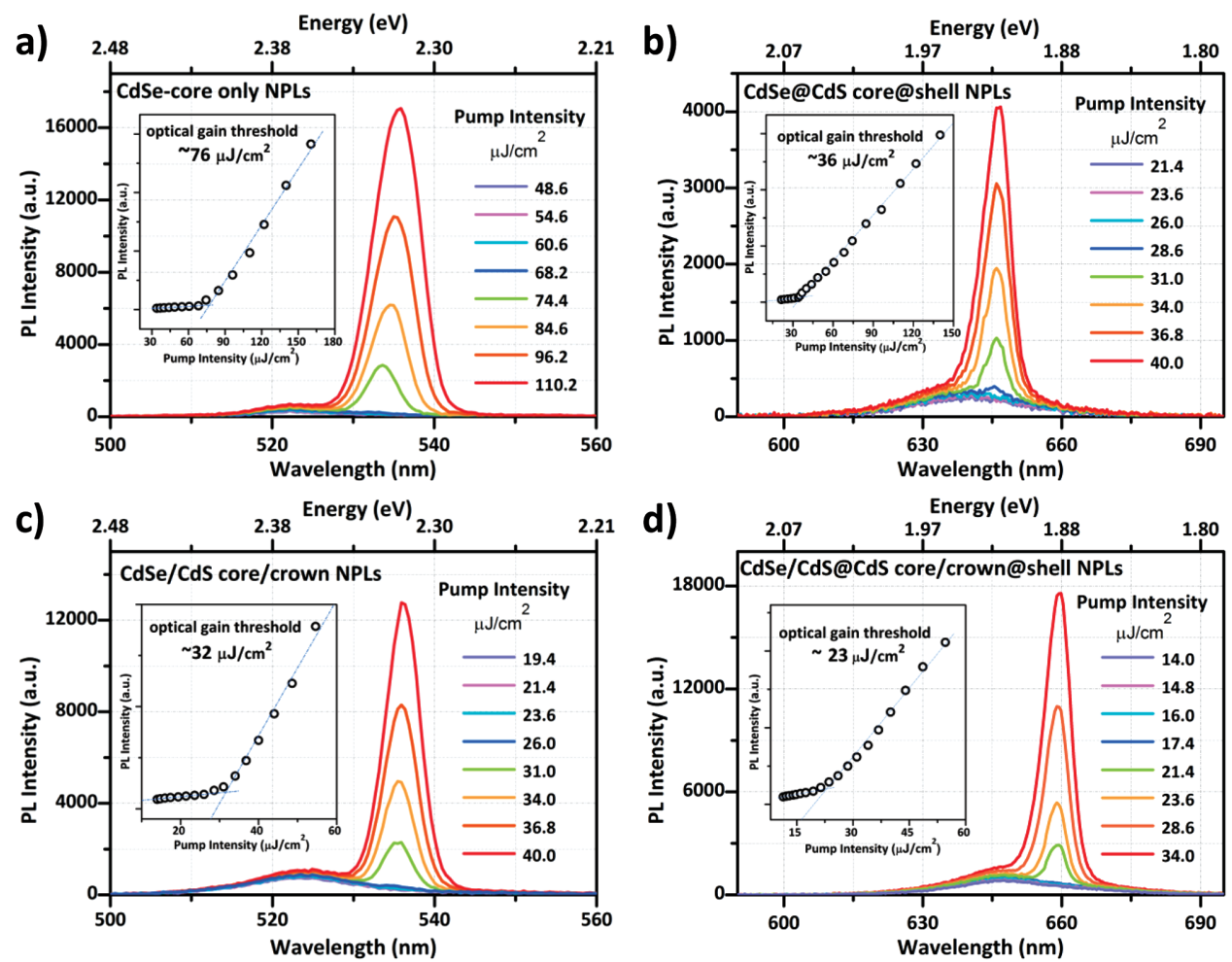

Figure 4. Optical gain performances of CdSe-based NPLs having different architectures. a) Amplified spontaneous emission (ASE) spectra of CdSe core-only NPLs, b) CdSe@CdS core@shell NPLs having 2 ML CdS-shell thickness, c) CdSe/CdS core/crown NPLs, and d) CdSe/CdS@CdS core/ crown@shell NPLs having 2 ML CdS-shell thickness. The insets show the integrated PL intensity as a function of the excitation intensity. 
CdSe core-only NPLs, when the excitation intensity exceeds

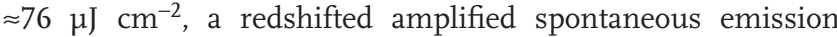
(ASE) spectrum is obtained. This redshifted ASE peak is previously observed in the CdSe core-only NPLs and attributed to the biexcitonic gain. ${ }^{[38,39]}$ Also, when compared to the previous reports on NPLs, our CdSe core-only NPLs exhibit slightly high gain thresholds. This is possibly due to the different NPL lateral sizes and qualities leading to different optical gain performances. ${ }^{[7]}$ Next, we studied optical gain performance of CdSe/ CdS C/C NPLs, which were synthesized by using the same CdSe core-only NPLs. With the formation of CdS-crown layer, we observed significantly decreased gain threshold (down

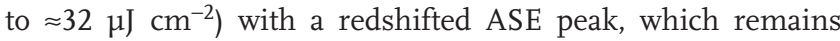
almost in the same spectral position compared to the core-only NPLs. The enhancement of the absorption cross-section in the C/C NPLs makes great contribution to help to decrease the gain threshold, which has been already demonstrated. ${ }^{[21]}$ Besides the increased absorption cross-section, the passivation of trap sites in the C/C NPLs results in higher optical quality and also helps to enhance optical gain performance.

To achieve further improved gain threshold, we studied the optical gain performance in the core@shell and core/crown@ shell NPLs having 2 ML CdS-shell thicknesses. With the formation of 2 ML CdS-shell layer, the CdSe@CdS core@shell NPLs exhibit lower gain threshold $\left(\approx 36 \mu \mathrm{J} \mathrm{cm} \mathrm{cm}^{-2}\right)$ with respect to the CdSe core-only NPLs. In this case, the lower gain threshold of the core@shell NPLs can be attributed to suppressed AR rates owing to quasi-Type-II electronic structure, where the electron and hole wavefunctions overlap is reduced. Also, the staircase-type relation between AR and NPL volume observed in the colloidal quantum wells, further helps with the suppression of AR in the C@S NPLs. ${ }^{[34]}$ Finally, with the combination of the enhanced absorption cross-section and the suppressed AR, we achieved the lowest gain threshold $\left(\approx 23 \mu \mathrm{J} \mathrm{cm} \mathrm{cm}^{-2}\right)$ from the CdSe/CdS@CdS core/crown@shell NPLs having 2 ML $\mathrm{CdS}$-shell. Here, the better peripheral passivation of the CdSe core-only NPLs in the C/C@S architecture also contributes to the decreased gain threshold, which results in high optical quality films and suppressed nonradiative energy transfer rates between the NPLs.

Figure 5 shows the intensity of the amplified spontaneous emission (ASE) in the core/crown@shell sample for pump laser shots up to $2.5 \times 10^{7}$ that correspond to overall $6 \mathrm{~h}$ of continuous excitation (pump laser is at $1 \mathrm{kHz}$ repetition rate). The excitation intensity was chosen to be $35 \mu \mathrm{J} \mathrm{cm}^{-2}$, which is above the ASE threshold of the sample $\left(\approx 20 \mu \mathrm{J} \mathrm{cm}^{-2}\right)$. ASE intensity shows a slight increase in the first $2 \mathrm{~h}$ of excitation, which we attribute to the annealing of the sample under the excitation condition due to slight heating up the sample. Almost after $6 \mathrm{~h}$ of continuous pumping, the ASE intensity drops just slightly. As compared to the CdSe core-only and CdSe/CdS core/crown NPLs, which have shown stable ASE only for several minutes of excitation $\left(\approx 1 \times 10^{6} \text { pump shots }\right)^{[19]}$ the core/crown@shell NPLs depict much greater stability. Thus, the core/crown@ shell architecture will be very promising to overcome the stability problems of the existing NPL architectures. Also, as compared to tailor-made core/shell quantum dots and nanorods with low gain, ${ }^{[35]}$ the stability of the ASE in the core/crown@ shell NPLs is better. Thanks to the complete passivation of the

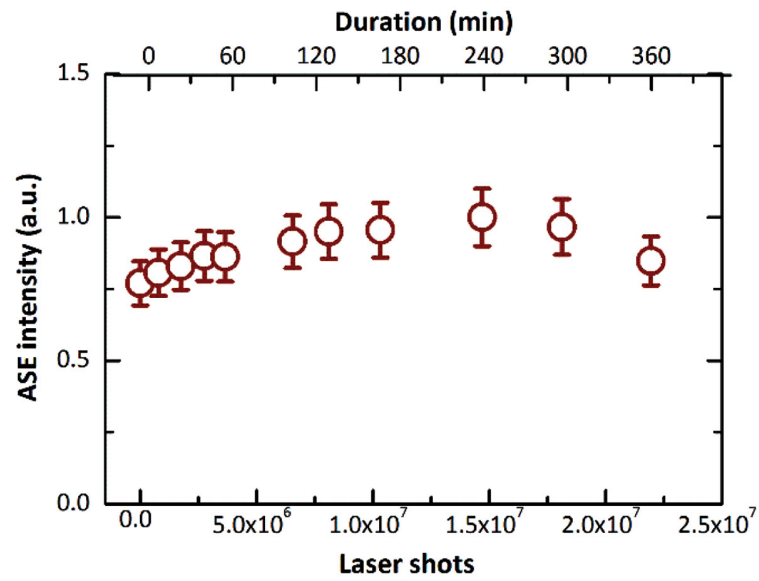

Figure 5. Intensity of the amplified spontaneous emission (ASE) in the core/crown@shell NPLs as a function of pump laser shots (per pulse energy density of $35 \mu \mathrm{cm}^{-2}$ ). The optical gain in the core/crown@shell NPLs is highly stable even for $6 \mathrm{~h}$ of continuous excitation.

structure with the crown and shell layers, excitons in the core/ crown@shell NPLs stay away from the potentially reactive surface traps or defects.

\section{Conclusion}

In conclusion, we have reported the first account of $\mathrm{CdSe} /$ CdS@CdS core/crown@shell NPLs having a novel 3D architecture resembling platelet-in-box heterostructure and studied their excitonic kinetics. Here, we have shown the critical importance of peripheral growth with laterally extending crown layer before the vertical shell growth to push the gain performance from NPLs. With the formation of CdS-crown layer covering around the periphery of the CdSe-core only NPLs, trap sites are strongly passivated in the CdSe/CdS@CdS C/C@S NPLs. Thus, these C/C@S NPLs exhibit systematically higher PL-QY when compared to the CdSe@CdS C@S NPLs regardless of the CdSe-core size, CdS-crown size, and CdS-shell thickness. In addition to the enhanced PL-QY, more symmetric emission behavior, along with suppressed lifetime broadening, observed in the CdSe/CdS@CdS C/C@S NPLs by decreasing temperature also confirms the effective passivation of these trap sites. In addition, when compared to the C@S NPLs, the C/C@S NPLs exhibit significantly enhanced absorption cross-section with reduced reabsorption effect. Enabled by these major advancements in the excitonic behavior, the proposed C/C@S NPLs exhibit the lowest gain threshold among different architectures of NPLs. These findings can provide an important guideline for the colloidal synthesis of highly efficient and stable NPLs, which can enable high-performance optoelectronic devices possibly challenging epitaxially-grown thin-film quantum well devices.

\section{Experimental Section}

Chemicals: Cadmium nitrate tetrahydrate $\left(\mathrm{Cd}\left(\mathrm{NO}_{3}\right)_{2} \cdot 4 \mathrm{H}_{2} \mathrm{O}\right)$ (99.999\% trace metals basis), sodium myristate (>99\%), technicalgrade 1-octadecene (ODE), selenium (Se) (99.999\% trace metals basis), 
sulfur (S) (99.998\% trace metals basis), cadmium acetate dihydrate $\left(\mathrm{Cd}(\mathrm{OAc})_{2} \cdot 2 \mathrm{H}_{2} \mathrm{O}\right)(>98 \%)$, technical-grade oleic acid (OA) $(90 \%)$, technical-grade oleylamine (OAm) (70\%), N-methylformamide (NMF) $(99 \%)$, and ammonium sulfide solution (40-48 wt\% in $\mathrm{H}_{2} \mathrm{O}$ ) were purchased from Sigma-Aldrich. Hexane, ethanol, methanol, toluene, and acetonitrile were purchased from Merck Millipore and used without any further purification.

Preparation of Cadmium Myristate: Cadmium myristate was prepared according to the recipe given in the literature. ${ }^{[14]}$ For a typical synthesis, $1.23 \mathrm{~g}$ of cadmium nitrate tetrahydrate was dissolved in $40 \mathrm{~mL}$ of methanol and $3.13 \mathrm{~g}$ of sodium myristate was dissolved in $250 \mathrm{~mL}$ of methanol. After complete dissolution of cadmium nitrate tetrahydrate and sodium myristate powders, solutions were mixed and stirred vigorously around $1 \mathrm{~h}$. Then, the cadmium myristate powders were precipitated by using centrifuge and dissolved in methanol. The washing step with methanol was followed at least three times to remove any unreacted and/or excess precursors. After successive washing steps, the precipitated part was completely dried under vacuum overnight.

Synthesis of the CdSe Core NPLs: CdSe nanoplatelets (NPLs) having four complete monolayers (4 ML) of CdSe with an additional layer of $\mathrm{Cd}$ atoms were synthesized with a slightly modified recipe from the literature. ${ }^{[14]}$ For a typical synthesis, $340 \mathrm{mg}$ of cadmium myristate, $24 \mathrm{mg}$ of Se and $30 \mathrm{~mL}$ of ODE were loaded into a $100 \mathrm{~mL}$ three-neck flask. The solution was degassed at $100{ }^{\circ} \mathrm{C}$ for an hour. After degassing step, the solution was heated to $240{ }^{\circ} \mathrm{C}$ under argon atmosphere. When the solution color becomes bright yellowish (generally around 180-200 ${ }^{\circ} \mathrm{C}$ ), $110-120 \mathrm{mg}$ of cadmium acetate dihydrate was swiftly added to the reaction solution. After 10 min growth at $240{ }^{\circ} \mathrm{C}, 1 \mathrm{~mL}$ of $\mathrm{OA}$ was injected and the temperature of the solution was decreased to room temperature. The cadmium precursor injection temperature and the growth time were found to be important for the resulting shape and size of CdSe core NPLs. After the purification step with ethanol, CdSe core NPLs are dissolved in hexane and stored for the subsequent CdScrown and CdS-shell growth.

Preparation of Anisotropic Growth Solution for CdS-Crown Region: Anisotropic growth solution was prepared following the previously published procedure with slight modifications. For the preparation of cadmium precursor, $480 \mathrm{mg}$ of cadmium acetate dihydrate, $340 \mu \mathrm{L}$ of $\mathrm{OA}$, and $2 \mathrm{~mL}$ of ODE were loaded in a three-neck flask. The solution was heated to $100{ }^{\circ} \mathrm{C}$ under ambient atmosphere with rigorous stirring and it was also regularly sonicated. Until the formation of whitish color homogeneous gel, heating and sonication steps were followed alternatingly. When the cadmium precursor was prepared, it was mixed with $3 \mathrm{~mL}$ of sulfur precursor (0.1 M S-ODE stock solution) and used for the CdS-crown coating.

Synthesis of $\mathrm{CdSe} / \mathrm{CdS}$ Core/Crown NPLs: For typical CdS-crown coating, $1 \mathrm{~mL}$ of CdSe core NPLs dissolved in hexane (having an optical density of $\approx 1$ at $350 \mathrm{~nm}$ ), $5 \mathrm{~mL}$ of ODE and $100 \mu \mathrm{L}$ of OA were loaded into a $50 \mathrm{~mL}$ three-neck flask. The solution was degassed at $100{ }^{\circ} \mathrm{C}$ for the complete removal of hexane, water, and any other organic solvents. After that, the solution was heated to $240{ }^{\circ} \mathrm{C}$ under argon flow for the coating of CdS-crown region. When the temperature reaches $240{ }^{\circ} \mathrm{C}$, certain amount of anisotropic growth mixture was injected at the rate of $8 \mathrm{~mL} \mathrm{~h}^{-1}$. After the injection of anisotropic growth mixture was finished, the resulting mixture was further annealed for $5 \mathrm{~min}$ at $240{ }^{\circ} \mathrm{C}$. Finally, the solution was cooled down to room temperature for the purification step. The as-synthesized $\mathrm{CdSe} / \mathrm{CdS}$ core/crown NPLs were precipitated by using ethanol. Finally, the precipitated NPLs were dissolved in hexane and stored for the following CdS-shell coating steps. According to the desired CdS-crown size, the injection amount of anisotropic growth mixture was tuned. Also, with the addition of $O A$ to the reaction mixture, more uniform CdS-crown growth with enhanced colloidal stability was achieved.

Synthesis of CdSe@CdS Core@Shell and CdSe/CdS@CdS Core/ Crown@Shell NPLs: BothCdSe@CdS C@S and CdSe/CdS@CdSC/C@S NPLs were synthesized by using the colloidal atomic layer deposition (c-ALD) technique. ${ }^{[20]} \mathrm{We}$ use CdSe-core only and $\mathrm{CdSe} / \mathrm{CdS}$ core/ crown NPLs as seeds for the subsequent CdS-shell coating. For a typical
CdS-shell deposition, $3 \mathrm{~mL}$ of $\mathrm{N}$-methylformamide (NMF) and $3 \mathrm{~mL}$ of seed NPLs dissolved in hexane were mixed. With the addition of $50 \mu \mathrm{L}$ of sulfur precursor (Ammonium sulfide solution), NPLs are transferred from hexane to NMF. After $5 \mathrm{~min}$ shaking and stirring for complete reaction, the NPLs were precipitated with the addition of acetonitrile and toluene. For complete removal of excess sulfur precursor, the washing step was repeated at least two times. Then, precipitated NPLs were dissolved in $3 \mathrm{~mL}$ of fresh NMF for the next cadmium deposition step. After complete dissolution of NPLs, $2 \mathrm{~mL}$ of cadmium precursor ( $0.2 \mathrm{M}$ cadmium acetate-NMF) was added and waited for $5 \mathrm{~min}$ to complete reaction. The NPLs were then precipitated with the addition of acetonitrile and toluene. Resultantly, $1 \mathrm{ML}$ CdS-shell was formed on the $\mathrm{CdSe}$-core or $\mathrm{CdSe} / \mathrm{CdS}$ core/crown NPLs. For further increasing the CdS-shell thickness, this process was followed in the same way. Finally, the core@shell and/or core/crown@shell NPLs with surfaces terminated with cadmium were precipitated and dispersed in hexane with the addition of excess amount of OAm.

For the TRF measurements, core-only, core/crown, core@shell, and core/crown@shell NPLs were precipitated with the addition of ethanol. Then, they were dissolved in hexane and the TRF measurements were conducted with the samples cleaned only one times to prevent removal of surface ligands and stacking issues, which significantly change the decay kinetics of NPLs. On the other hand, for the optical gain study, we performed the same washing procedure with ethanol at least two times for the achievement of highly close packed and uniform films form NPLs.

\section{Supporting Information}

Supporting Information is available from the Wiley Online Library or from the author.

\section{Acknowledgements}

The authors acknowledge the financial support from Singapore National Research Foundation under the programs of NRF-RF-2009-09 and NRF-CRP-6-2010-02 and the Science and Engineering Research Council, Agency for Science, Technology and Research ( $*$ *STAR) of Singapore; EU-FP7 Nanophotonics4Energy NoE; and TUBITAK EEEAG 114E449 and 114F326. H.V.D. acknowledges support from ESF-EURYI and TUBAGEBIP. Y.K., K.G., and O.E. acknowledge support from TUBITAK BIDEB.

Received: February 2, 2016

Revised: February 26, 2016

Published online: April 13, 2016

[1] C. B. Murray, D. J. Norris, M. G. Bawendi, J. Am. Chem. Soc. 1993, 115,8706

[2] A. P. Alivisatos, X. Peng, L. Manna, W. Yang, J. Wickham, E. Scher, A. Kadavanich, Nature 2000, 404, 59.

[3] L. Manna, E. C. Scher, A. P. Alivisatos, J. Am. Chem. Soc. 2000, 122, 12700 .

[4] S. Ithurria, B. Dubertret, J. Am. Chem. Soc. 2008, 130, 16504.

[5] S. Ithurria, M. D. Tessier, B. Mahler, R. P. S. M. Lobo, B. Dubertret, A. L. Efros, Nat. Mater. 2011, 10, 936.

[6] E. Lhuillier, S. Pedetti, S. Ithurria, B. Nadal, H. Heuclin, B. Dubertret, Acc. Chem. Res. 2015, 48, 22.

[7] M. Olutas, B. Guzelturk, Y. Kelestemur, A. Yeltik, S. Delikanli, H. V. Demir, ACS Nano 2015, 9, 5041.

[8] A. Yeltik, S. Delikanli, M. Olutas, Y. Kelestemur, B. Guzelturk, H. V. Demir, J. Phys. Chem. C 2015, 119, 26768. 
[9] R. Xie, U. Kolb, J. Li, T. Basché, A. Mews, J. Am. Chem. Soc. 2005, $127,7480$.

[10] D. V Talapin, J. H. Nelson, E. V Shevchenko, S. Aloni, B. Sadtler, A. P. Alivisatos, Nano Lett. 2007, 7, 2951.

[11] S. Jana, T. N. T. Phan, C. Bouet, M. D. Tessier, P. Davidson, B. Dubertret, B. Abécassis, Langmuir 2015, 31, 10532.

[12] B. Abécassis, M. D. Tessier, P. Davidson, B. Dubertret, Nano Lett. 2014, 14, 710

[13] B. Guzelturk, O. Erdem, M. Olutas, Y. Kelestemur, H. V. Demir, ACS Nano 2014, 8, 12524

[14] M. D. Tessier, P. Spinicelli, D. Dupont, G. Patriarche, S. Ithurria, B. Dubertret, Nano Lett. 2014, 14, 207.

[15] A. Prudnikau, A. Chuvilin, M. Artemyev, J. Am. Chem. Soc. 2013 135,14476

[16] S. Delikanli, B. Guzelturk, P. L. Hernández-Martínez, T. Erdem, Y. Kelestemur, M. Olutas, M. Z. Akgul, H. V Demir, Adv. Funct. Mater. 2015, 25, 4282.

[17] S. Pedetti, S. Ithurria, H. Heuclin, G. Patriarche, B. Dubertret, J. Am. Chem. Soc. 2014, 136, 16430.

[18] Y. Kelestemur, M. Olutas, S. Delikanli, B. Guzelturk, M. Z. Akgul, H. V. Demir, J. Phys. Chem. C 2015, 119, 2177.

[19] B. Mahler, B. Nadal, C. Bouet, G. Patriarche, B. Dubertret, J. Am. Chem. Soc. 2012, 134, 18591.

[20] S. Ithurria, D. V Talapin, J. Am. Chem. Soc. 2012, 134, 18585.

[21] B. Guzelturk, Y. Kelestemur, M. Olutas, S. Delikanli, H. V. Demir, ACS Nano 2014, 8, 6599

[22] M. D. Tessier, B. Mahler, B. Nadal, H. Heuclin, S. Pedetti, B. Dubertret, Nano Lett. 2013, 13, 3321.

[23] L. T. Kunneman, M. D. Tessier, H. Heuclin, B. Dubertret, Y. V Aulin, F. C. Grozema, J. M. Schins, L. D. a Siebbeles, J. Phys. Chem. Lett. 2013, 4, 3574

[24] Z. Chen, B. Nadal, B. Mahler, H. Aubin, B. Dubertret, Adv. Funct. Mater. 2014, 24, 295
[25] C. She, I. Fedin, D. S. Dolzhnikov, A. Demortière, R. D. Schaller, M. Pelton, D. V Talapin, Nano Lett. 2014, 14, 2772.

[26] B. Guzelturk, M. Olutas, S. Delikanli, Y. Kelestemur, O. Erdem, H. V. Demir, Nanoscale 2015, 7, 2545

[27] M. D. Tessier, C. Javaux, I. Maksimovic, V. Loriette, B. Dubertret, ACS Nano 2012, 6, 6751

[28] L. T. Kunneman, J. M. Schins, S. Pedetti, H. Heuclin, F. C. Grozema, A. J. Houtepen, B. Dubertret, L. D. a Siebbeles, Nano Lett. 2014, 14, 7039.

[29] K. E. Knowles, E. a. McArthur, E. a. Weiss, ACS Nano 2011, 5, 2026

[30] P. Jing, J. Zheng, M. Ikezawa, X. Liu, S. Lv, X. Kong, J. Zhao, Y. Masumoto, J. Phys. Chem. C 2009, 113, 13545.

[31] J. Li, K. B. Nam, J. Y. Lin, H. X. Jiang, Appl. Phys. Lett. 2001, 79, 3245.

[32] L. Grenouillet, C. Bru-Chevallier, G. Guillot, P. Gilet, P. Duvaut, C. Vannuffel, a. Million, a. Chenevas-Paule, Appl. Phys. Lett. 2000, 76, 2241.

[33] J. Munguía, J.-M. Bluet, O. Marty, G. Bremond, M. Mermoux, D. Rouchon, Appl. Phys. Lett. 2012, 100, 102107.

[34] C. She, I. Fedin, D. S. Dolzhnikov, P. D. Dahlberg, G. S. Engel, R. D. Schaller, D. V Talapin, ACS Nano 2015, 9, 9475.

[35] B. Guzelturk, Y. Kelestemur, K. Gungor, A. Yeltik, M. Z. Akgul, Y. Wang, R. Chen, C. Dang, H. Sun, H. V. Demir, Adv. Mater. 2015, 27, 2741.

[36] J. Q. Grim, S. Christodoulou, F. Di Stasio, R. Krahne, R. Cingolani, L. Manna, I. Moreels, Nat. Nanotechnol. 2014, 9, 891.

[37] B. Guzelturk, Y. Kelestemur, M. Z. Akgul, V. K. Sharma, H. V. Demir, J. Phys. Chem. Lett. 2014, 5, 2214.

[38] Y. Kelestemur, A. F. Cihan, B. Guzelturk, H. V. Demir, Nanoscale 2014, 6, 8509.

[39] A. F. Cihan, Y. Kelestemur, B. Guzelturk, O. Yerli, U. Kurum, H. G. Yaglioglu, A. Elmali, H. V. Demir, J. Phys. Chem. Lett. 2013, $4,4146$. 